\title{
Single Photon Communication with Avalanche Diodes and the General Basics of Photon Counting
}

\author{
Boldizsár KURILLA ${ }^{1}$
}

\begin{abstract}
Single photon communication (SPC) already exists in several applications in laboratory and even outdoor conditions. In the field of quantum cryptography SPC experiments are part of military applications too. There are several methods to detect every single impacting photon in such an experiment. Mostly photomultiplier tubes (PMT) are used. In some cases single photon avalanche diodes (SPAD) are more suitable for photon detection. Both the SPADs and PMTs have advantages and disadvantages. Usually PMTs have much larger detection areas than SPADs, but most of the PMTs detection efficiency peaks at $400 \mathrm{~nm}$ wavelength compared to the SPADs, where it peaks at 600-700 nm wavelength. For long distance laser measurements the higher wavelength is more suitable due to the Rayleigh scattering, but the detection hole of SPAD is very tight, which is why it is really hard to target the laser punctually without an optical gyroscope.
\end{abstract}

Keywords: Avalanche diode, single photon communication, photomultiplier tube, dead time, noise, Photon Detection Efficiency (PDE)

\section{Introduction}

In general, light detection is possible by the measurement of electrical signals induced by the absorption of the certain light. Photonic communication is based on the modulation of light coming into a detector. There are many ways to modulate light for communication purposes. Meanwhile the single photon communication opportunities open many ways for the safety processes in the field of laser communication experiments. It was demonstrated and published previously that single photon communication is possible in outdoor conditions even over very large distances. [1] [6] Photon counting is possible with the help of a PMT and a photon counter such as the SR400 in Second Harmonic Generation experiments which was already demonstrated in laboratory conditions. [2] The other possible way is to use an optical delay system with an impulse generator and a wavelength doubled laser for PMTs or a red laser for SPADs. Originally the optical delay system was used to develop the antibunching photon. [3] In our case the current detector system is mainly based on the APDs and SPADs instead of PMTs. The use of SPAD based devices has already proved to be remarkably advantageous in a few applications. [23]

It was found from the measurement of the tracking time of pulses and the number of pulses observed per unit time that the number of photoelectrons considered as probability are variable. This can be described by mathematical statistics and the means of probability

1 E-mail: kurilla.boldizsar@gmail.com 
calculations. Electrical impulses received during the detection - a seemingly obvious assumption - is matched to the individual photons. Comparing this with the intensity of classic light, it can be concluded that in a specific location $(r)$ and on a certain $d A$ surface under unit time a photon detection probability $p(r)$ is proportional to the intensity $I(r)$ of light (in the classical sense):

$$
P(r) \times d A \approx I(r) \times d A
$$

The detection of single electrons from the viewpoint of experimental physics is a technical question, which needs to be handled correctly (the coincidence multi-photonic, multi-electronic pulses, dead time, noise, etc.). [5]

\section{Digital Signal Processing Methods, Photon Counting and the Features of Photon Counter Detectors}

The pulse given by the multiplication of a single photoelectron can be handled as digital sign. However, if it is expected to be handled in the usual digital device system, it will not be fulfilled yet, unfortunately. There are several reasons why it will not work:

1. The amplitude of the pulses are not the same.

2. The width of the pulses is influenced by many parameters.

3. The dead time, the maximum repetition frequency can also be taken into account.

4. For the examination of the temporal behavior it has to be standardized that when shall be considered a real photon to be "arrived".

The parameters of the two basic types of photon counting detector (APD and the PMT) are different: If we talk about the amplitude at PMT, then we have to realize that the noise and distribution for each photoelectron overlap each other, but only with a partially selectable amplitude discriminator (with the exclusion of very small amplitude - but prolific - noise pulses).

At APDs in Geiger mode (avalanche range) the noise and the photoelectron generate a pulse form. Here it is not possible to select.

About pulse width, it must be pointed out that a PMT works with 50 Ohm load in order to avoid reflections on the cable. Those pulses are dynode capacity dependent which are 1-2 ns width at half maximum.

At APDs there is a 5-10 ns signal falloff after 2 ns upraising. [5]

In Figure 1 the amplitude distribution of the PMT pulses can be seen. 
Boldizsár KURILLA: Single Photon Communication with Avalanche Diodes and the General Basics...

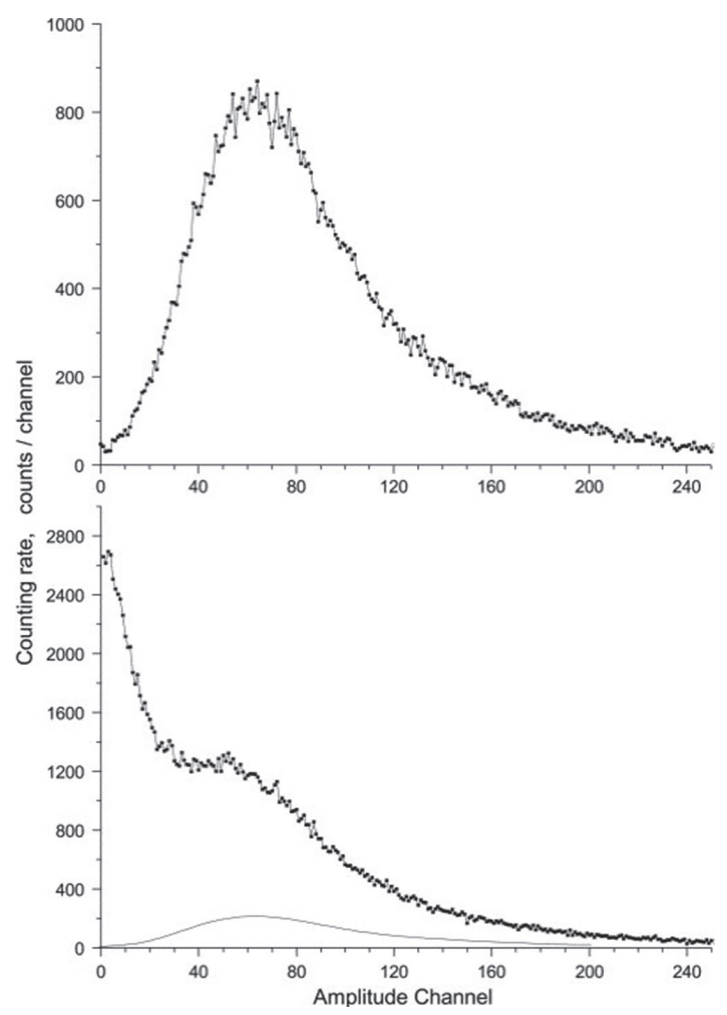

Figure 1. Amplitude distribution of the PMT pulses. (At the bottom with low noise, at the top the single electron distribution given by the subtraction of noise.) [5]

In Figure 2 the oscilloscope view of pulses of avalanche photodiode matrix (M-APD) can be seen. At the bottom the dark noise pulses, at the top the photoelectric pulses can be seen, separable from the $1,2,3, \ldots$ pixel simultaneous sounds evoked impulses.

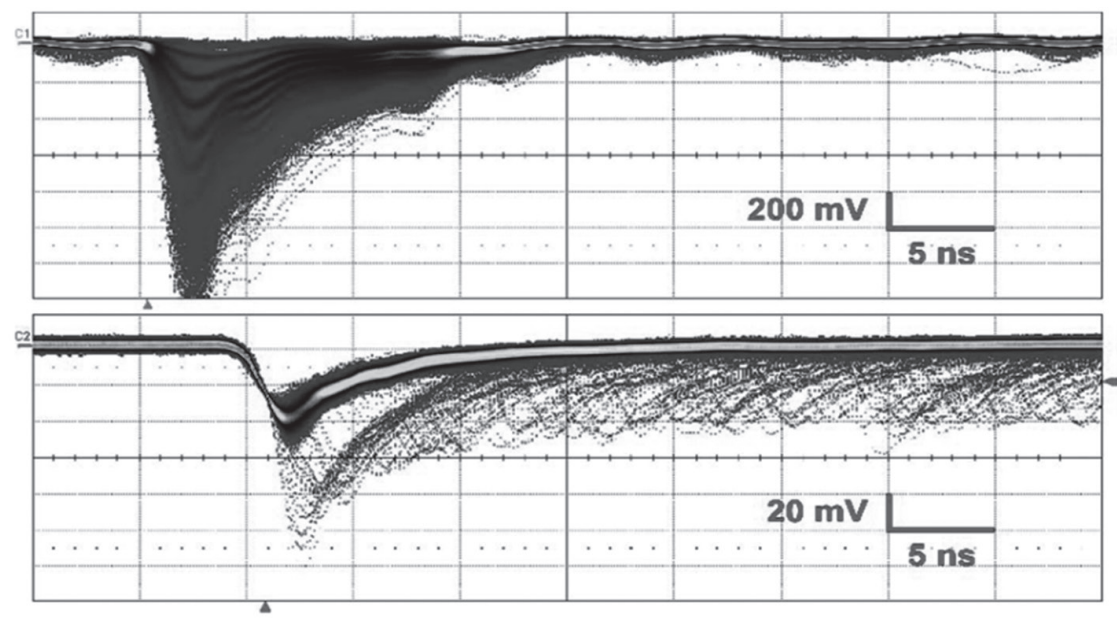

Figure 2. Oscilloscope view of pulses of avalanche photodiode matrix (M-APD). [5] 
The parameters of the two detectors (PMT and Avalanche photodiodes (APDs) are different. In terms of amplitude of APD we cannot select in Geiger mode (avalanche range) where the noise and the photoelectron generates a pulse form. In terms of amplitude of PMT, the noise and the distribution belonging to the individual photoelectrons overlap each other, but the amplitude is partially selectable with the help of a discriminator. In this case the low amplitude, but prolific noise pulses must be excluded.

To increase the maximum count rate of a PMT based SPC system it is practical to utilize multiple discriminators. [20] PMTs of different designs and operation principles have been introduced already based on vacuum-tube technology. Those classes of PMTs exhibit narrower height distributions than those of metal-dynode PMTs. [21]

The avalanche photodiodes are very fast acting tools (1-2 ns), and these are also suitable for the registration of photoelectrons raised by individual photons. It has a proportional range too depending on the high voltage, but it is mostly used in photon counting (Geiger-Müller) mode. That means that the formation of one electron-hole pair results in a complete short-circuit. Similarly to the working of the ionization detector, it needs time for the recovery of pre-voltage (dead time). This limits the maximum measurement frequency. It has been already recorded that at a certain wavelength range with this tool the quantum efficiency reached above $90 \%$ as well. [5] Figure 3 shows the APD pulses relative to the time sliding of light pulse and the full width at half maximum of the scatter is $210 \mathrm{ps}$.

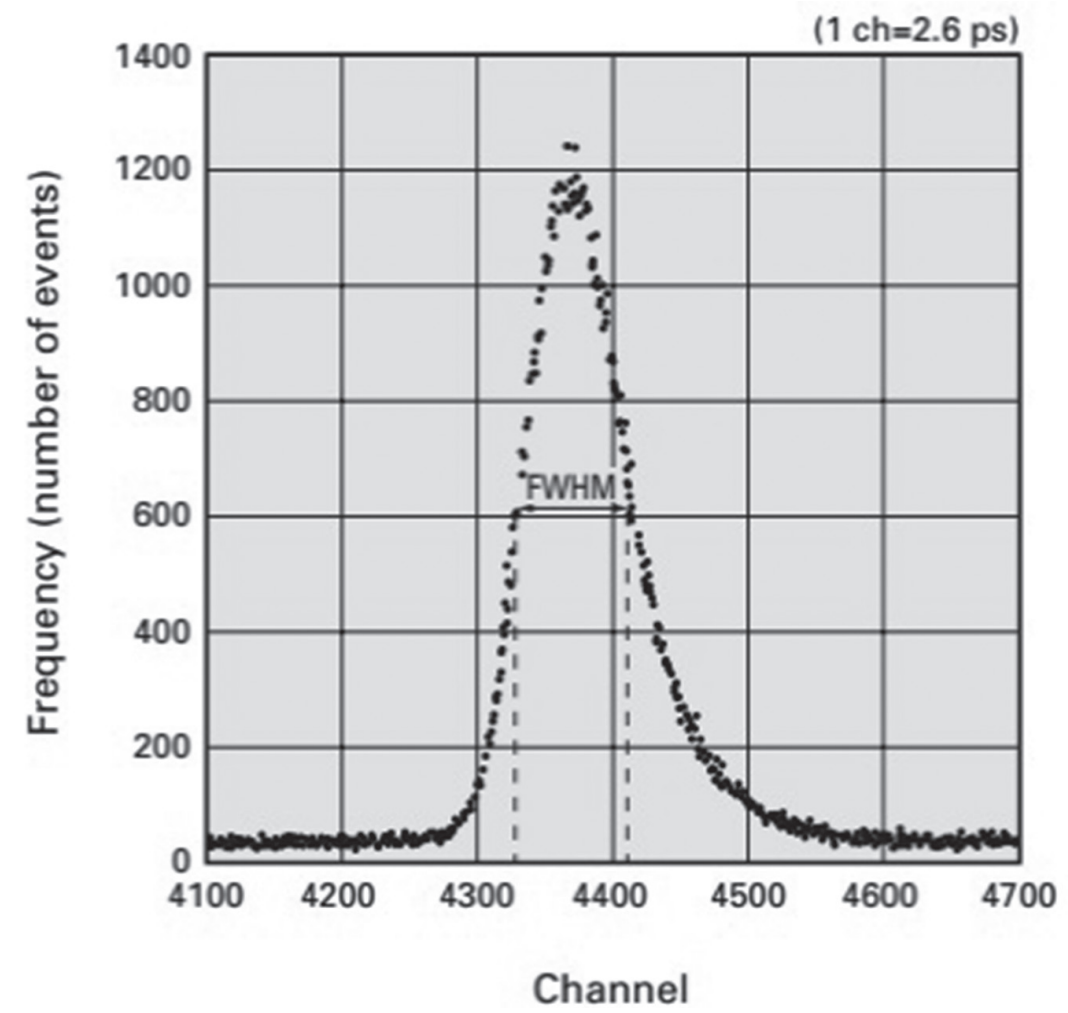

Figure 3. Avalanche photodiode (APD) pulses relative to the time sliding of light pulse (jitter), the full width at half maximum of the scatter. [5] 
The principles of time correlated SPC is to detect less than one photon many times repeatedly per cycle. One of the biggest advantages of single photon counting is that it can be used in a really wide range of light intensity:

1. The upper limit is given by the pulse width or dead time (approximately 190-200 MHz).

2. The lower limit is namely the noise fluctuation (approximately 20 pulses/s).

The quotient of these two limits is called dynamic range: $\mathrm{D}=\mathrm{Imax} / \mathrm{Inoise}$, which is given by either logarithmic or exponential - performance being $10^{1}=20 \mathrm{~dB}$ - unit form. In our case the performance is typically $10^{7}=140 \mathrm{~dB}$. Another similar dynamic analog detector does not exist (the typical value is three orders of magnitude). [5] Picture 1 shows the amplitude fluctuation and the distribution function of a single photoelectron signal.

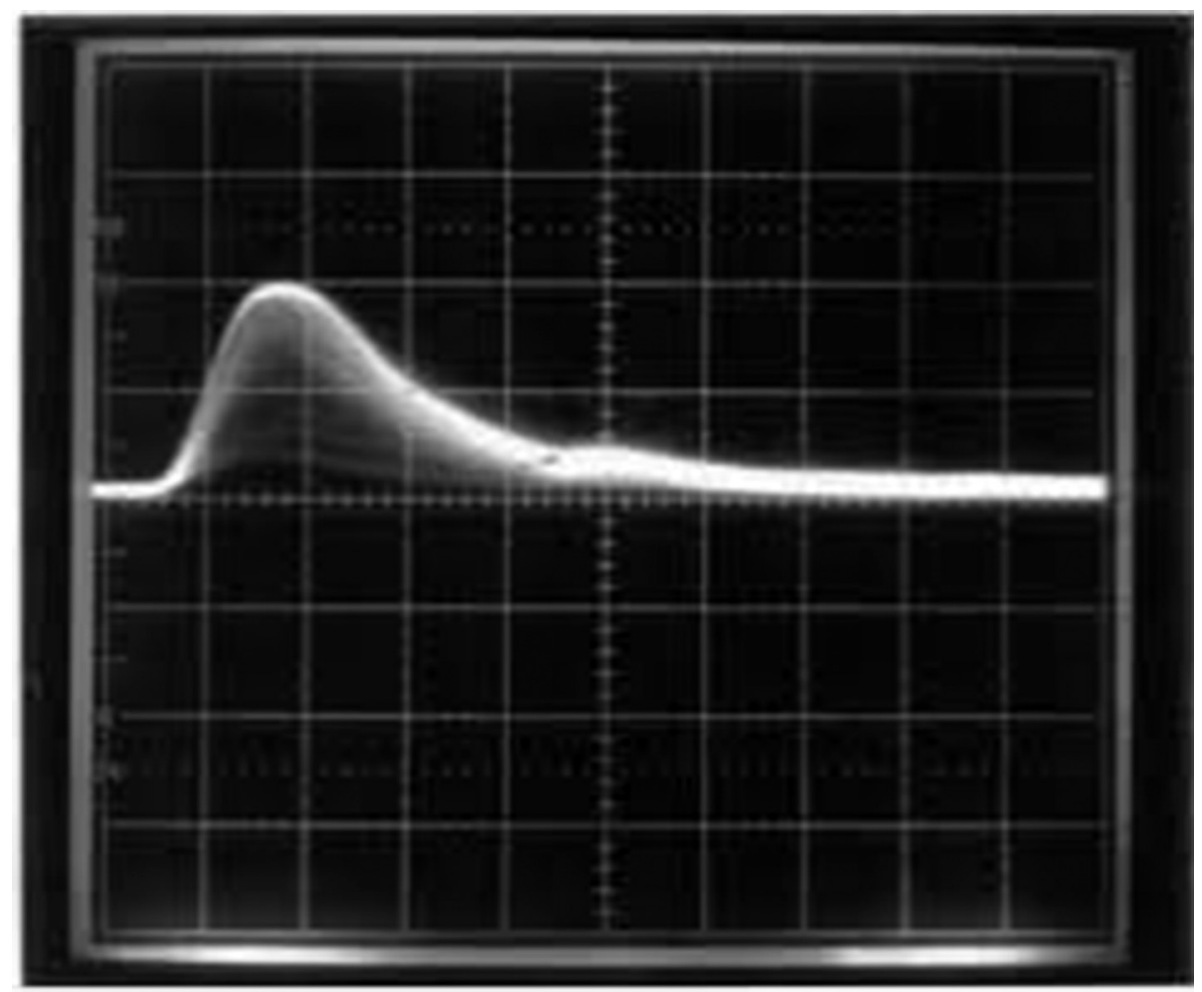

Picture 1. The amplitude fluctuation and the distribution function of a photoelectron signal. [5]

The basics of photon counting has been discussed, [1] [2] but here it is also important to understand the basics of gated photon counting to get a more intense introduction to the photonic communication procedure. This will help us to understand the basics of avalanche diodes. 


\section{Gated Photon Counting}

To digitally process the signal of the photon counter detectors, they are needed to bring them to the signal level of the usual logic circuits. Typically, this means the level of integrated circuit components made by Complementary Metal-Oxide Semiconductor (CMOS) technique: in the family of $+5 \mathrm{Vs}$ the voltage signal ranging is between 0 and $+1.5 \mathrm{~V}$ at the Low level range, and it can be between $3.5+\ldots+5 \mathrm{~V}$ at the High level range. It shall be chargeable, although in the older TTL family the consumption of one input was only $40 \mu \mathrm{A}$. The CMOS circuits may be perceived more as a capacitive load, so it is only to be expected at high frequencies. To count many photons, short pulses (1-2 ns) should be juggled from our detector, and this is already showing that the wave phenomena will spread in the cables between the devices. If it does not meet with the purely resistive impedance in accordance with its wave impedance, then it will be reflected. For this reason, the detector usually works at 50 Ohm resistor (as a power generator), and the electronic load is not significant. For this reason, the detector usually works for 50 Ohm resistor (as a power generator), and the electronic load is not significant. The problem is that the detector can emit only 10-100 mV amplitude pulses. In order to reach the standard level of these logic circuits, it needs to be reinforced, or with a help of a comparator transform the signals to rectangular shape signals. The gain is not really feasible, as the amplitude (PMT) often shows an order of magnitude deviation. If we want to count only the "photons", a so called fast comparator is used, pulses above the set level generate a quick uprising, the required $5 \mathrm{~V}$ amplitude square signal. The comparator is often referred to as a discriminator. In fact, by an adequate setting of comparison threshold the small amplitude noise pulses can be ruled out (discriminate). Often upper level discriminators are also used, since the pulses induced by cosmic rays are much larger than the single photon signals. This type is called window discriminator. The pulse width is influenced by two conflicting considerations:

1. The processing electronics - counter - speed, that is what minimum pulse width react to (the older, slower devices meant large capacitive load, only adequately wide pulse was able to fill the capacitor of the input stage).

2. If we have a modern counter, then the shorter the output pulses are, the less dead time is needed to count, that we can measure higher intensity more frequently. The $200 \mathrm{MHz}$ counters are frequent, and that is why the photocurrent is needed to be converted to less than 5ns pulse.

3. If we use longer dead-time APDs, we do not need an extremely fast counter, because the detector would not be able to indicate frequently (maximum $10 \mathrm{MHz}$ ). [5]

In Figure 4 different types of photon counting techniques can be seen. From the view of photon counting the typical optical spectroscopic measurements that require time resolution fall into three main categories:

There can be less than 1 photon per cycle in the periodic measurement cycles. It needs bigger time resolution than what the detector has (to all the time windows impact only one photon once in 10-100 cycles).

There are two photons in one cycle and the time resolution has a greater scale than the temporal resolution of the detector (one cycle of time window contains a lot of photos).

There are many photons in one cycle and the temporal position of each photon can be fixed by adjusting the definition of the detector (in one cycle to a time window impacts only one photon). [5] 


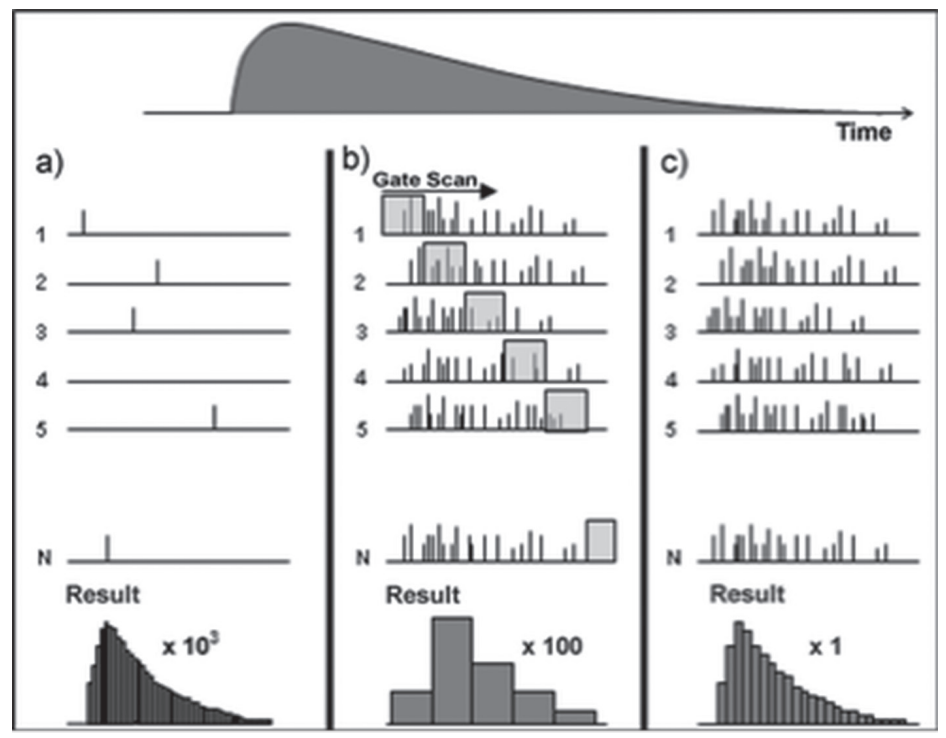

Figure 4. Various techniques for single photon counting. [5]

The name of Version A. Time Correlated Single Photon Counting. It outlines the case when a very fast process takes place after the short excitation pulse. It needs to have better time resolution than most of the detectors have (1-2 ns). This can be achieved by a certain theoretical agreement that explains that the detection probability of classical light intensity and the certain photon in time unit - proportional to each other - by definition.

So if the detectors are unsuitable for breaking down the simultaneously arriving photons on the chosen time scale, then it must be achieved that there should be only one photon at the time of a measurement cycle. The temporal distance of the detection and appearance of photon will be the parameter (measured from the short excitation impulse), which will be measured. The processing system uses an appropriate number of time channels. All measurements give a time frame, it will be the time channel number. All contents corresponding to the cycle time channel measurement results will increase the value by 1 . After many measurement cycles $\left(10^{5}-10^{6}\right)$ the probability density function of the photons temporal distribution emerges. The figure of this is equal to the time function, which could have been measured as analog signal by a detector with proper resolution. It is obligatory to make very low intensity signal response for the measurement. It can be achieved by intentional depletion. Preferably it can be achieved by the weakening of excitation, but the response signal can be also weakened with the gap narrowing of the spectral resolution, or with the use of gray filter. Since the detection of photons is a random process, we must investigate what are the chances of arrival not only for one photon in a measurement cycle to the detector, but two or more. Specifically, we must determine the average intensity of what comes to the detector which in the case of the simultaneous presence of a sufficient number of photons have low probability. [5]

Version B.: gated integration. In this version the gate width is much longer than the time dissolution of the detector and this steps forward in time with a gate width relative to the exciting pulse in every cycle. For this we need only one single-channel counter, performing data processing unit (PC) is organizing the scanning time. 
Version $C$. is supposed to be a multi-channel counter (PC with a digital I/O/timer/counter card), where every measurement cycle are the same. The measurement must go on until there will be enough counts for the statistical processing in the individual channels.

\section{Principles of SPADs}

SPAD detectors so far reported can be divided in two groups, according to the depletion layer of the p-n junction, which can be thin, typically $1 \mu \mathrm{m}$, [10] [11] or thick, from $20 \mu \mathrm{m}$ to $150 \mu \mathrm{m}$. [7] [8] [9] [12] [13] [14] However the required active area at the largest fast SPADs have a diameter with even $200 \mu \mathrm{m}$. The main features of thin-junction SPADs are: fairly good quantum efficiency in the visible range, about $45 \%$ at $500 \mathrm{~nm}$ which declines to $32 \%$ at $630 \mathrm{~nm}$ and to $15 \%$ at $730 \mathrm{~nm}$, and is still useful in the near infrared (NIR), being about $10 \%$ at $830 \mathrm{~nm}$ and a few $0.1 \%$ at $1064 \mathrm{~nm}$; small active area, with diameter from $20 \mu \mathrm{m}$ to $100 \mu \mathrm{m}$; breakdown voltage $\mathrm{VB}$ of $20 \mathrm{~V}-50 \mathrm{~V}$. Instead thick-junction silicon SPADs have: breakdown voltage VB of 200V-500V; fairly wide active area, with diameter from $100 \mu \mathrm{m}$ to $500 \mu \mathrm{m}$; quantum efficiency that is very high in the visible region, remarkably better than $50 \%$ over the range from $540 \mathrm{~nm}$ to $850 \mathrm{~nm}$ wavelength, and declines in the NIR, but is still about $3 \%$ at $1064 \mathrm{~nm}$. [4]

Figure 5 shows the cross-section of a thick SPAD. [19] The active area is defined by an $\mathrm{n}++$ phosphorous diffusion both by increasing the $\mathrm{p}-$ quasi-intrinsic substrate concentration with a p diffusion and by fabricating low-doped $n-$ phosphorous guard rings at the junction edges. The wafer is then flipped and back etched down to 30-40 $\mu \mathrm{m}$ from the active area. A $\mathrm{p}^{++}$boron diffusion is then created to provide a low resistance path for the avalanche current and a good ohmic contact with the anode metal deposition. The backside contact is the cathode. This structure is designed to deplete all the 30-40 $\mu \mathrm{m}$ thickness, thus providing both a thick depletion region and a high electric field all over the space-charge zone. [4]

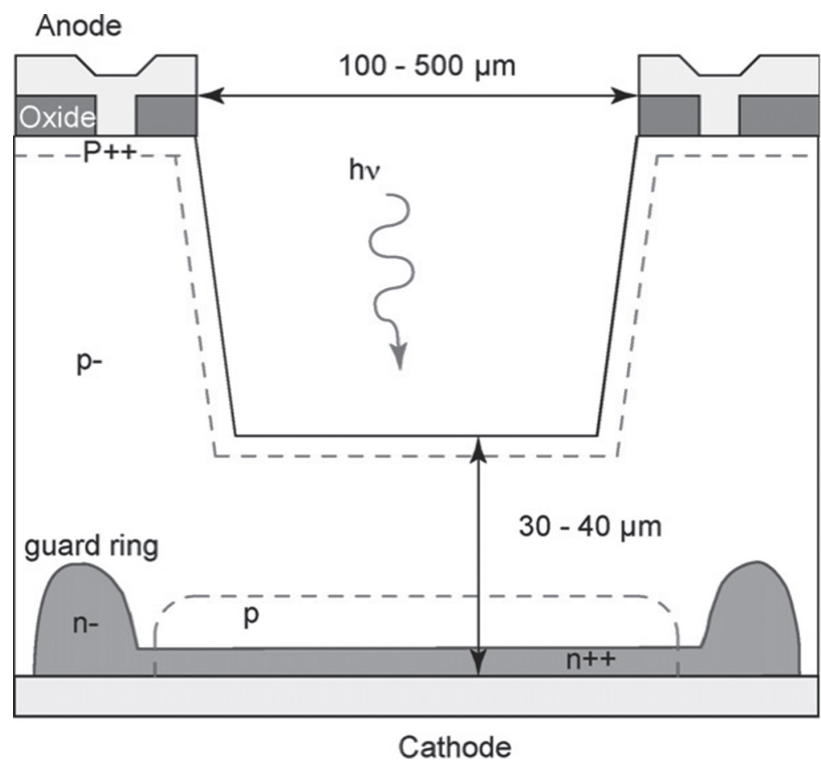

Figure 5. Cross-section of a thick SPAD. [4] 


\section{Difference between APDs and SPADs}

The term SPAD defines a specifically designed category of APDs working with a reverse bias well above the breakdown voltage, in a way that completely differs from normal APDs, operated below the breakdown level. The reverse biased p-i-n diodes are common silicon photodetectors essentially. That means the incident light generates electron-hole pairs in the depletion region contributing to the reverse current. The increase of the diode current is proportional to the incident light intensity. In order to have an internal gain between absorbed photons and output carriers, many APDs were proposed and developed. [15] They operate just near, but below breakdown which means that photo-generated carriers can produce other carriers via impact ionization process (Figure 6 on the left). However such process is not diverging and the result is an amplified response compared to normal photodetectors. [4]
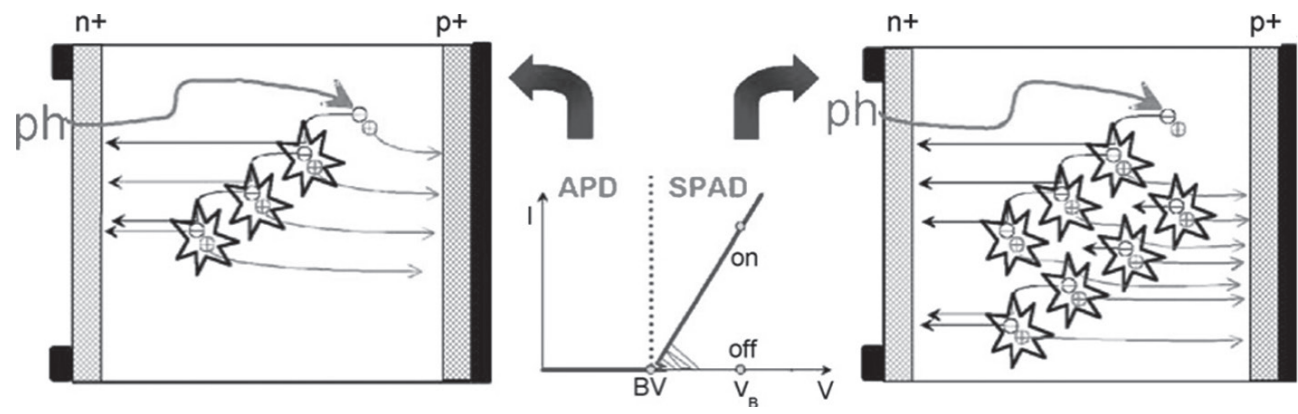

Figure 6. APDs are biased just below VB in order to have a non-diverging multiplication process (linear multiplication) of the photo-generated carriers (left side).

Instead SPADs are biased well above VB so that one photo-generated carrier can trigger a diverging avalanche multiplication process, leading to a macroscopic detectable output current (right side). [4]

Because the avalanche process has high statistical fluctuations (leading to excess noise [18]), APDs are used with relatively low gain (a few hundred at best), and therefore the detection of single-photons is possible just in few cases, with severe limitations and low detection efficiency. Single-photon avalanche diodes (SPADs) exploit avalanche multiplication in a different way. At a bias higher than the breakdown voltage, they work in Geiger-mode (Figure 6 on the right): a photo-generated carrier in the depletion region can trigger a diverging avalanche multiplication of carriers by impact ionization. [15] [17] Impact ionization involves both positive and negative carriers, with an inherent positive feedback effect that, if the electric field is high enough, making the carrier multiplication self-sustaining. This is often referred to as a divergence to infinity of the multiplication factor; in fact, the current is finite because of a space-charge effect, [15] which produces a finite internal resistance of the device that lowers the voltage drop across the junction. In normal APDs, turning off the incident light will immediately terminate the multiplication. Instead, an SPAD does not turn off by itself when triggered, - therefore the photo-diode must be reset, i.e. the avalanche process must be quenched -, in order to make the detection of a subsequent photon possible. [4] 
Figure 7 shows an example of the overall photon-detection efficiency as a function of the excess bias for thin and thick SPADs and Figure 8 shows the photon detection efficiency (PDE) of thick, thin and Red-Enhanced SPADs.
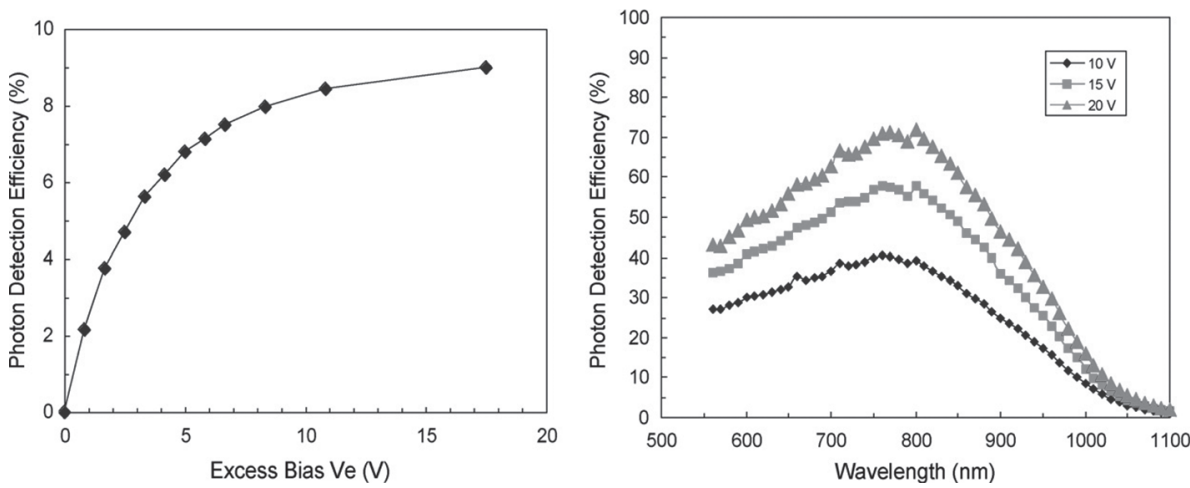

Figure 7. Photon-detection efficiency at different excess bias for a thin $\operatorname{SPAD}(W=1 \mu \mathrm{m})$ at $\lambda=850 \mathrm{~nm}$ (left) and for a thick SPAD (W=25 $\mu \mathrm{m})$ (right). [4] [16]

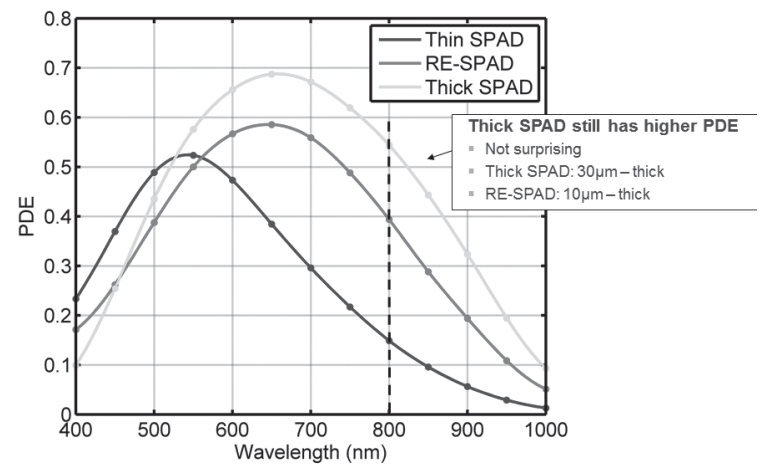

Figure 8. PDE of thick, thin and Red-Enhanced SPADs. [22]

\section{Conclusions}

In this paper the basics of photon counting and the basic principles of single photon avalanche diodes and avalanche photo diodes have been discussed. APD and SPAD characteristics and physics have been also discussed and analyzed. As we have seen there are various techniques for single photon counting, where all methods have advantages, but technological difficulties too. The question of "which one we want to use?" depends on what we want to communicate and of course also depends on the technological instruments we have. For outdoor measurements during daylight it is extremely difficult to use APDs and SPADs for single photon communication due to the very high sensitivity of these detectors. Even in a very dark night we can have difficulties due to the light of the stars. To make outdoor measurements with these tools one of the acceptable steps is to use high quality filters on the front of detectors. 
Boldizsár KURILLA: Single Photon Communication with Avalanche Diodes and the General Basics...

\section{References}

[1] URSIN, R., TIEFENBACHER, F., SCHMITT-MANDERBACH, T., WEIER, H., SCHEIDL, T., LINDENTHAL, M., BLAUENSTEINER, B., JENNEWEIN, T., PERDIGUES, J., TROJEK, P., ÖMER, B., FÜRST, M., MEYENBURG, M., RARITY, J., SODNIK, Z., BARBIERI, C., WEINFURTER, H., ZEILINGER, A.: Free-Space distribution of entanglement and single photons over $144 \mathrm{~km}$. Ithaca: Cornell University, 2007. www.arxiv.org/pdf/quant-ph/0607182 (downloaded: 1109 2015)

[2] KURILLA B.: Second Harmonic Generation in the Background of Photon Counting. AARMS, 134 (2014), 557-570.

[3] KURILLA B.: A lézerek alapjai, lézerfizikai eszközök és alkalmazásaik robottechnikai eszközökön. Hadmérnök, IX 1 (2014), 217-230. www.hadmernok.hu/141_21_kurillab.pdf (downloaded: 1109 2015)

[4] ZAPPA, F., TISA, S., TOSI, A., COVA, S.: Principles and features of single-photon avalanche diode arrays. Sensors and Actuators, A 140 (2007), 103-112.

[5] SÁNTA I.: Optoelectronics. TÁMOP-4.1.2.A/1-11/1, 2011. (MSc-tananyagfejlesztés) (downloaded: 0910 2015)

[6] KURILLA B.: Lézeres kommunikációt befolyásoló légköri tényezők vizsgálata szimulációs módszerrel. Hadmérnök, IX 2 (2014), 297-310.

[7] DAUTET, H., DESCHAMPS, P., DION, B., McGREGOR, A. D., McSWEEN, D., McINTRY, R. J., TROTTIER, C., WEBB, P. P.: Photon counting techniques with silicon avalanche photodiodes. Applied Optics, 32 (1993), 3894-3900. http://physics.ucsd. edu/ tmurphy/apollo/doc/Dautet.pdf (downloaded: 2510 2015)

[8] SPCM-AQ Single-photon counting module data sheet. Perkin Elmer Optoelectronics, 2005. http://opto.perkinelmer.com (downloaded: 11.11.2015)

[9] GHIONI, M., RIPAMONTI, G.: Improving the performance of commercially available Geiger-mode avalanche photodiodes. Review of Scientific Instruments, 62 (1991), 63-167.

[10] COVA, S., LACAITA, A. L., GHIONI, M., RIPAMONTI, LOUIS, T. A.: 20-ps timing resolution with single-photon avalanche diodes. Review of Scientific Instruments, 60 (1989), 1104-1110.

[11] LACAITA, A., GHIONI, M., COVA, S.: Double epitaxy improves single-photon avalanche diode performance. Electronics Letter, 2513 (1989), 841-843.

[12] BROWN, R. G., RIDLEY, K. D., RARITY, J. G.: Characterization of silicon avalanche photodiodes for photon correlation measurements. 1: Passive Quenching. Applied Optics, 25 (1986), 4122-4126. DOI: https://doi.org/10.1364/AO.25.004122

[13] BROWN, R. G., JONES, R., RARITY, J. G., RIDLEY, K. D.: Characterization of silicon avalanche photodiodes for photon correlation measurements. 2: Active Quenching. Applied Optics, 26 (1987), 2383-2389. DOI: https://doi.org/10.1364/AO.26.002383

[14] LI, L. M., LI-QUIANG, D.: Single photon avalanche diode for single molecule detection. Review of Scientific Instruments, 64 (1993), 1524-1529.

[15] SZE, S. M.: Physics of Semiconductor Devices. New York: J. Wiley and Sons, 1981.

[16] COVA, S., GHIONI, M., LACAITA, A., SAMORI, C., ZAPPA, F.: Avalanche photodiodes and quenching circuits for single-photon detection. Applied Optics, 3512 (1996), 19561976. DOI: https://doi.org/10.1364/AO.35.001956

[17] GROVE, A. S.: Physics and Technology of Semiconductor Devices. New York: J. Wiley and Sons, 1967. 
Boldizsár KURILLA: Single Photon Communication with Avalanche Diodes and the General Basics...

[18] McINTYRE, R.: On the avalanche initiation probability of avalanche diodes above the breakdown voltage. IEEE Electron Device Letters, 2020 (1973), 637-641. DOI: https:// doi.org/10.1109/T-ED.1973.17715

[19] McINTYRE, R. J., WEBB, P. P.: Low-noise, reach-through avalanche photodiodes. US Patent 5583352A. New York: United States Patent and Trademark Office, 1996.

[20] SOUKKA, J., VIRKKI, A., HÄNNINEN, P., SOINI, J.: Optimization of multi-photon event discrimination levels using Poisson statistics. Optics Express, 12 (2004), 84-89. DOI: https://doi.org/10.1364/OPEX.12.000084

[21] PERKIN ELMER OPTOELECTRONICS: Channel Photomultipliers - New Technology for More Accurate and Efficient Photon Detection. http://optoelectronics.perkinelmer.com/ content/WhitePapers/WTP_CPMPhotonCounting.pdf (downloaded: 1411 2015)

[22] COVA, S., GHIONI, M., ZAPPA, F., RECH, I., TOSI, A., GULINATTI, A.: SPADLab presentation. Milano: Politecnico de Milano, 2013. http:/home.deib.polimi.it/cova/elet/ Articoli\%20e\%20presentazioni/2013SPADlab_SSN.pdf (downloaded: 1511 2015)

[23] PANZERI, F., INGARGIOLA, A., LIN, R. R., SARKHOSH, N., GULINATTI, A., RECH, I., GHIONI, M., COVA, S., WEISS, S., MICHALET, X.: Single-molecule FRET experiments with a red-enhanced custom technology SPAD. 2013. www.ncbi.nlm.nih.gov/ pmc/articles/PMC3872070/ (downloaded: 1011 2015) 